\title{
Measurement of Second Hyperpolarizability and Nuclear Rotational Response of Gas-phase Carbon Disulfide
}

\author{
Peng Zhao, Matthew Reichert, David J. Hagan and Eric W. Van Stryland* \\ CREOL, The College of Optics and Photonics, University of Central Florida, Orlando, FL, 32816, USA \\ *ewvs@creol.ucf.edu
}

\begin{abstract}
We use nonlinear beam deflection to separate the bound-electronic and nuclearrotational responses in gas-phase $\mathrm{CS}_{2}$. This allows a direct comparison of the second hyperpolarizability to our liquid $\mathrm{CS}_{2}$ measurements considering local-field effects.

OCIS codes: (190.0190) Nonlinear optics, (190.3270) Kerr effect, (190.5650) Raman effect, (350.3250) Isotope separation
\end{abstract}

\section{Introduction}

The nonlinear optical (NLO) response of a gaseous medium under an intense laser field has been extensively studied and knowledge of the precise magnitudes and temporal dynamics of the third-order nonlinear refraction (NLR) are critical for various applications including filamentation. The total NLR response of gas-phase molecules excited by an ultrashort pulse are governed by a nearly instantaneous response that originates from the bound-electronic second hyperpolarizability, and a non-instantaneous response from field-induced nuclear rotation due to two-photon stimulated Raman transitions [1]. As a widely used NLO standard material, carbon disulfide $\left(\mathrm{CS}_{2}\right)$ has been thoroughly studied in its liquid form, where the major NLR responses are characterized by a bound-electronic Kerr index $n_{2, e l}$ plus three major nuclear contributions [2]. The macroscopic $n_{2, e l}$ is related to the real part of the orientationally averaged microscopic second hyperpolarizability $\gamma_{\mathrm{s}}$ via the molecular number density $N$ and a thirdorder Lorentz local-field factor $f^{(3)}$ by eq. (1) [4], where $c$ is the speed of light in vacuum, $\varepsilon_{0}$ is the vacuum permittivity, and $n_{\mathrm{e}}$ and $n_{p}$ are the real linear refractive index of excitation and probe respectively.

$$
\operatorname{Re}\left[\gamma_{s}\right]=\frac{4 \varepsilon_{0}^{2} c n_{e} n_{p}}{3 N f^{(3)}} n_{2, e l},
$$

An approach to measuring the hyperpolarizability without the need for local-field corrections is to directly measure nonlinear refraction in the gas phase where negligible local-field effects are present $\left(f^{(3)} \sim 1\right)$. In this work, we expand upon our recently developed beam deflection technique [3] to measure NLR transients of gas-phase $\mathrm{CS}_{2}$ using femtosecond pulses. The bound-electronic and nuclear rotation responses are separated, allowing an unambiguous determination of the $\operatorname{Re}\left[\gamma_{s}\right]$, which makes possible direct comparison to the $\operatorname{Re}\left[\gamma_{s}\right]$ obtained from our liquid-phase measurements [2].

\section{Experiment and Results}

The beam deflection technique uses a focused strong excitation beam to induce a refractive index change in the sample that follows its spatially Gaussian irradiance profile [2,3]. The probe pulse is focused $\sim 5 \times$ smaller and is displaced to where the index gradient is largest, as shown in Fig. 1a. The refractive index gradient induced by the excitation acts as a prism deflecting the probe beam by a small angle. The $\mathrm{CS}_{2}$ vapor (Sigma-Aldrich, 270660, $\geq 99.9 \%$ purity) is contained in a $100 \mathrm{~mm}$ path length cell such that the beams to not overlap at the cell windows. The beam crossing geometry is illustrated in Fig. 1b, where the excitation crosses the probe in the vertical (yz) plane, but is displaced in the horizontal (xz) plane, as shown in Fig. 1c, causing deflection in the x-direction. In this experiment we use $\sim 10 \mathrm{~mJ}, \sim 40 \mathrm{fs}(\mathrm{FWHM})$ pulses at a $1 \mathrm{kHz}$ repetition rate from a Ti:Sapphire amplified laser system (Coherent Legend Elite Duo $\mathrm{HE}+$ ) to pump an optical parametric amplifier (TOPAS-HE) to produce excitation pulses at $1250 \mathrm{~nm}$. A portion of the excitation is used to generate a white-light continuum (WLC) in a 5 $\mathrm{mm}$ thick sapphire plate, which is then spectrally filtered by bandpass interference filters $(\Delta \lambda=10 \mathrm{~nm}$ FWHM) at $950 \mathrm{~nm}$ to use as probe. The probe is detected by a quad-segmented Si photodiode (OSI QD50-0-SD) placed in the far-field. The difference in energy falling on the left and right sides of the segmented photodiode as shown in Fig. 1c $\Delta E=E_{\text {left }}-E_{\text {right }}$, is measured using lock-in detection and normalized to the total energy $E$, yielding the deflection signal $\Delta E / E$. In the small signal limit, $\Delta E / E$ is proportional to the refractive index change $\Delta n$ [3]. By temporally delaying the excitation and probe, NLR transients can be resolved, and by varying the polarization angle between the excitation and probe, the tensor symmetry of the nonlinear susceptibility is measured. At the magic angle $\left(\sim 54.7^{\circ}\right)$ the nuclear rotation response is eliminated, which isolates the bound-electronic NLR and enables direct measurement of $\operatorname{Re}\left[\gamma_{s}\right]$.

Fig. 1d shows the measured $\Delta E / E$ of gas-phase CS2 with its polarization dependence, which consists of an instantaneous bound-electronic response $\left(\Delta n_{e l}\right)$ at zero delay and a slowly rising rotational response $\left(\Delta n_{\text {rot }}\right)$ with a 

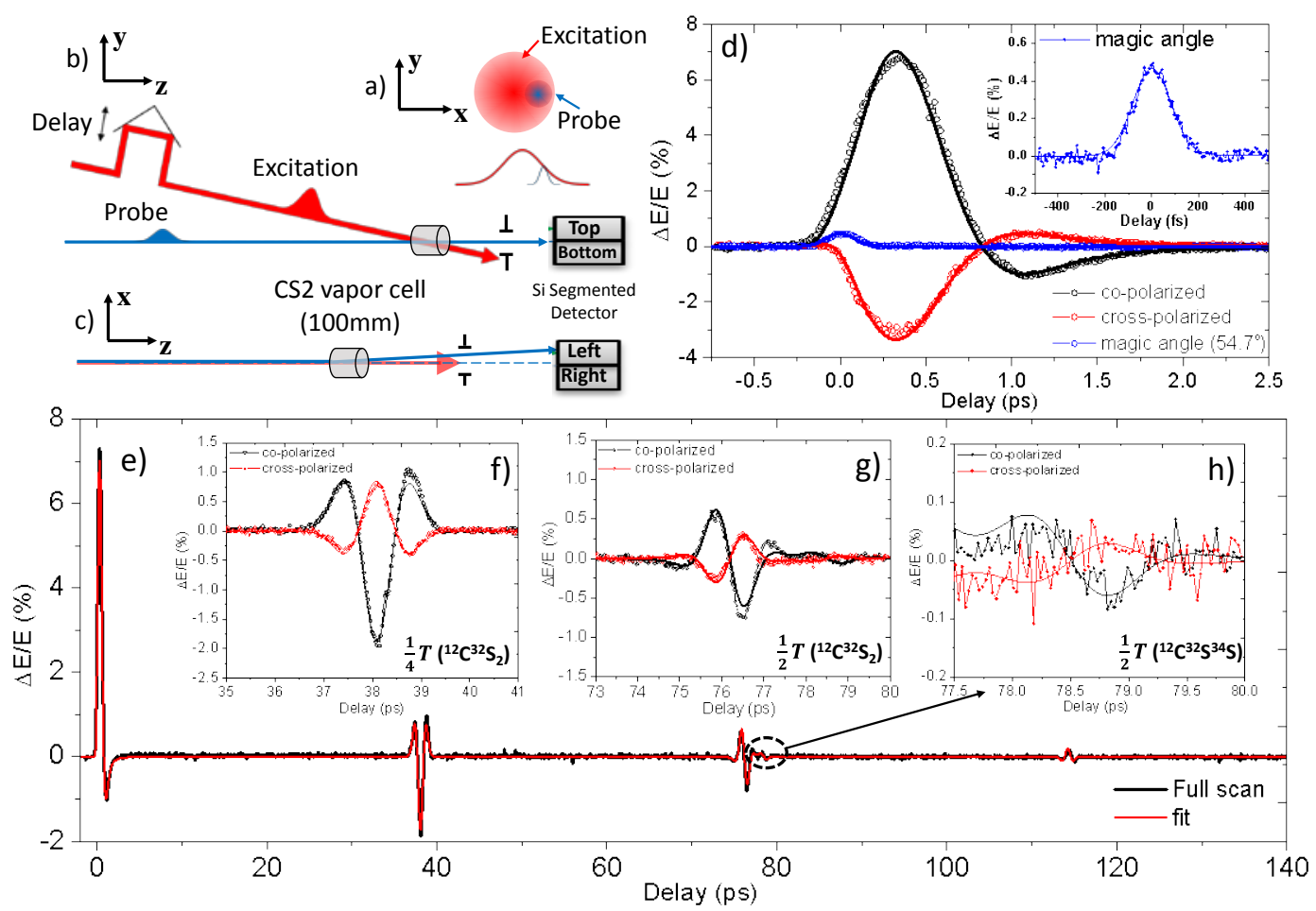

Fig. 1. (a-c) Excitation and probe overlap geometry in beam deflection measurement of gas-phase $\mathrm{CS}_{2}$; (d) Polarization dependence of measured (circles) and fit (curves) signal at zero delay (inset shows enlarged magic angle measurement); (e) NLR response of $\mathrm{CS}_{2}$ vapor with co-polarized excitation and probe measured up to 140 ps. The inset show $1 / 4(\mathrm{f}), 1 / 2(\mathrm{~g})$ revivals from ${ }^{12} \mathrm{C}^{32} \mathrm{~S}_{2}$ and ${ }^{1 / 2}(\mathrm{~h})$ revival from isotopologue ${ }^{12} \mathrm{C}^{32} \mathrm{~S}^{34} \mathrm{~S}$.

maximum at $\sim 400 \mathrm{fs}$. The rotational response arises from the excitation induced net alignment of the molecular ensemble via Raman transitions. For polarization parallel // and perpendicular $\perp$ to the molecular axis of $\mathrm{CS}_{2}$ with polarizability anisotropy $\Delta \alpha=\alpha / /-\alpha_{\perp}$, the excitation pulse creates birefringence when the molecules align towards the excitation polarization direction (i.e. < 400fs in Fig. 1d). Here the co- and cross-polarized probe will experience positive and negative $\Delta n_{\text {rot }}$ respectively, following the reorientational symmetry $\Delta n_{\text {rot }, \perp}=-\Delta n_{\text {rot, }, / / 2}$. At the magic angle the rotational component is eliminated $\left(\Delta n_{\text {rot }}=0\right)$. This separation of $\Delta n_{e l}$ and $\Delta n_{\text {rot }}$ enables the determination of the $\operatorname{Re}\left[\gamma_{s}\right]$ through a relative measurement. With the well-known $\Delta \alpha\left(10.53 \times 10^{-40} \mathrm{Fm}^{2}\right)$ of $\mathrm{CS}_{2}$ molecules from Ref. [5], we use the temporally delayed rotational response $\left(\Delta n_{\text {rot }} \propto N(\Delta \alpha)^{2}\right)$ as a reference to measure the boundelectronic response $\left(\Delta n_{e l} \propto N \operatorname{Re}\left[\gamma_{s}\right]\right)$ along with $\Delta n_{\text {rot }}$ in a self-consistent measurement, where the measured magnitude of $\Delta n_{e l} / \Delta n_{\text {rot }}$ is proportional to $\operatorname{Re}\left[\gamma_{s}\right] /(\Delta \alpha)^{2}$ [1, 3]. Following the isotropic tensor symmetry $\Delta n_{e l, \perp}=\Delta n_{e l, / l}$ / 3, we can fit $\Delta n_{e l}$ of the gas-phase $\mathrm{CS}_{2}$ measurements for co-, cross- and magic-angle polarization data with $\operatorname{Re}\left[\gamma_{s}\right]($ vapor $)=(1.8 \pm 0.4) \times 10^{-61} \mathrm{C}^{4} \mathrm{~m}^{4} \mathrm{~J}^{-3}\left((1.5 \pm 0.3) \times 10^{-36} \mathrm{esu}\right)$, which is slightly smaller than (but within errors of $)$ the $\operatorname{Re}\left[\gamma_{s}\right]$ (liquid) $=(2.2 \pm 0.5) \times 10^{-61} \mathrm{C}^{4} \mathrm{~m}^{4} \mathrm{~J}^{-3}\left((1.8 \pm 0.4) \times 10^{-36}\right.$ esu) calculated considering local-field effects using $f^{(3)} \sim 5.3$ and $n_{2, e l}=(1.6 \pm 0.3) \times 10^{-19} \mathrm{~m}^{2} / \mathrm{W}$ from our liquid $\mathrm{CS}_{2}$ measurements [2]. The NLR transients of $\mathrm{CS}_{2}$ vapor are also measured with co-polarized beams for longer delays, where we observe the typical rotational revivals up to 3 quarter multiples of the revival period ( $T \sim 153 \mathrm{ps}$ ), as shown in Fig. 1e with fine structure at $1 / 4 T, 1 / 2 T$ revivals in the insets, which correspond to a rotational constant of $B\left({ }^{12} \mathrm{C}^{32} \mathrm{~S}_{2}\right)=0.1093 \pm 0.0002 \mathrm{~cm}^{-1}$. Interestingly, the signal at $\sim 79$ ps turns out to be the first half revival from the isotopologue ${ }^{12} \mathrm{C}^{32} \mathrm{~S}^{34} \mathrm{~S}$ (Fig. 1h), which can be fit by assuming the natural abundance of ${ }^{34} \mathrm{~S}(4.25 \%)$ and ${ }^{32} \mathrm{~S}(95 \%)$ [6] with $B\left({ }^{12} \mathrm{C}^{32} \mathrm{~S}^{34} \mathrm{~S}\right)=0.1061 \pm 0.0008 \mathrm{~cm}^{-1}$.

\section{Conclusion}

The second hyperpolarizability of $\mathrm{CS}_{2}$ is directly measured in gas-phase via the ultrafast nonlinear beam deflection method and is approximately equal to that measured in the liquid phase including local field effects. $\operatorname{Re}\left[\gamma_{s}\right]$ of gasphase molecules is of interest for comparison to theoretical models. By resolving the rotational response of ${ }^{12} \mathrm{C}^{32} \mathrm{~S}^{34} \mathrm{~S}$, beam deflection shows a potential application for isotope identification.

\section{Reference}

1. $\quad$ Lin, C.H., et al., Physical Review A, 1976. 13(2): p. 813-829.

2. $\quad$ Reichert, M., et al., Optica, 2014. 1(6): p. 436-445.

3. Ferdinandus, M.R., et al., Optics Letters, 2013. 38(18): p. 3518-3521.

4. $\quad$ Armstrong, J.A., et al., Physical Review, 1962. 127(6): p. 1918-1939.

5. Bogaard, M.P., et al., Journal of the Chemical Society, 1978. 74(0): p. 3008-3015.

6. The Atomic Weights of the Elements: Review 2000 (IUPAC Technical Report), IUPAC. 2003. p. 21. 